\title{
The role of angular ordering condition in Parton Branching transverse momentum dependent (TMD) PDFs and DY transverse momentum spectrum at LHC
}

\author{
Aleksandra Lelek* \\ UAntwerp \\ E-mail: aleksandra.lelek@uantwerpen.be
}

We discuss the parton branching (PB) evolution equation for transverse momentum dependent (TMD) parton distribution functions (PDFs), especially we concentrate on the angular ordering constrain and its effect on the TMD distributions.

We discuss application of the PB TMDs to precise prediction of Drell-Yan transverse momentum spectrum at LHC, stressing the role of angular ordering in the low transverse momentum region. We compare the PB implementation of the angular ordering condition with the Kimber-MartinRyskin-Watt (KMRW), both at the analytical and numerical level.

European Physical Society Conference on High Energy Physics - EPS-HEP2019 -

10-17 July, 2019

Ghent, Belgium

${ }^{*}$ Speaker. 


\section{Why TMDs?}

Collinear factorization theorem [1] is a successful tool commonly used to obtain QCD predictions for sufficiently inclusive collider observables with only one hard scale relevant. However, for observables with a second scale involved (as e.g. transverse momentum in the final state) the collinear approximation is not sufficient and transverse momentum dependent (TMD) factorization theorems should be used to obtain precise predictions [2].

One of the open problems is a consistent kinematics treatment in parton showers (PS). As reported in [3], when the PS is added on top of the collinear matrix element (ME), the energymomentum conservation requires that the kinematics in the ME must be manipulated to compensate for the transverse momentum generated in the PS procedure. This leads to a mismatch between the 4 momenta of the partons in the ME and the parton distribution functions (PDFs) initially used for the generation. Based on the TMD factorization [4], an approach was proposed [5] where off-shell (TMD) ME could be used together with TMD PDFs (abbreviated here as TMDs). Higher order QCD corrections could be included via TMD PS. Thanks to this procedure, the transverse momentum generated in the ME would not be changed by adding the PS and the kinematics would be properly treated. The key ingredient to make this approach possible is the availability of the TMDs.

The Parton Branching (PB) method developed in the last few years [6, 7] is an approach which allows one to obtain TMDs. The method incorporates the phenomena of angular ordering $[8,9]$ - the fact that the colour coherence effects result in certain ordering in angle of the soft gluons emitted in QCD radiation. In the following we examine the role of the angular ordering condition in obtaining precise QCD predictions for LHC observables, concentrating especially on the scale dependent soft gluon resolution parameter which was extensively studied in [10].

\section{Angular ordering in PB}

The PB TMD evolution equation with angular ordering has the following form [7]

$$
\begin{aligned}
& \widetilde{A}_{a}\left(x, \boldsymbol{k}, \mu^{2}\right)=\Delta_{a}\left(\mu^{2}, \mu_{0}^{2}\right) \widetilde{A}_{a}\left(x, \boldsymbol{k}, \mu_{0}^{2}\right)+\sum_{b} \int \frac{\mathrm{d}^{2} \boldsymbol{\mu}^{\prime}}{\pi \mu^{\prime 2}} \Theta\left(\mu^{2}-\mu^{\prime 2}\right) \Theta\left(\mu^{\prime 2}-\mu_{0}^{2}\right) \\
\times & \int_{x}^{1-\frac{q_{0}}{\mu^{\prime}}} \mathrm{d} z \frac{\Delta_{a}\left(\mu^{2}, \mu_{0}^{2}\right)}{\Delta_{a}\left(\mu^{\prime 2}, \mu_{0}^{2}\right)} P_{a b}^{R}\left(z, \alpha_{s}\left((1-z)^{2} \mu^{\prime 2}\right)\right) \widetilde{A}_{b}\left(\frac{x}{z}, \boldsymbol{k}+(1-z) \boldsymbol{\mu}^{\prime}, \mu^{\prime 2}\right),
\end{aligned}
$$

where $\widetilde{A}_{a}\left(x, \boldsymbol{k}, \mu^{2}\right)=x A_{a}\left(x, \boldsymbol{k}, \mu^{2}\right)$ is the momentum-weighted TMD distribution of a parton of flavor $a$, carrying the longitudinal momentum fraction $x$ of the proton's momentum and transverse momentum $\boldsymbol{k}^{1}$ at the evolution scale $\mu ; z$ and $\boldsymbol{\mu}^{\prime}$ are the branching variables, with $z$ being the ratio of the proton's longitudinal momentum fraction carried by parton $a$ and $b$, and $\mu^{\prime}=\sqrt{\boldsymbol{\mu}^{\prime 2}}$ the scale at which the branching happens. $P_{a b}^{R}$ are the real-emission splitting functions and $\Delta_{a}$ is the Sudakov form factor, given by $\Delta_{a}\left(\mu^{2}, \mu_{0}^{2}\right)=\exp \left[-\sum_{b} \int_{\mu_{0}^{2}}^{\mu^{2}} \frac{\mathrm{d} \mu^{\prime 2}}{\mu^{\prime 2}} \int_{0}^{1-q_{0} / \mu^{\prime}} \mathrm{d} z z P_{b a}^{R}\left(z, \alpha_{s}\left((1-z)^{2} \mu^{\prime 2}\right)\right)\right]$. The initial evolution scale is denoted by $\mu_{0}$. The parameter $q_{0}$ defining the soft gluon resolution scale,

\footnotetext{
${ }^{1}$ The following notation is used: $k=\left(k^{0}, k^{1}, k^{2}, k^{3}\right)=\left(E_{k}, \boldsymbol{k}, k^{3}\right)$, where $\boldsymbol{k}=\left(k^{1}, k^{2}\right)$, and $k_{\perp}=|\boldsymbol{k}|$.
} 
is the minimum transverse momentum of the emitted parton, with which it can be resolved. The dynamical resolution scale $z_{M} \equiv 1-\frac{q_{0}}{\mu^{\prime}}$ depends on the scale of the branching $\mu^{\prime}$. Eq. (2.1) defines also the relation between the transverse momentum of the emitted parton and the scale at which the branching happens $q_{\perp}=(1-z) \mu^{\prime}$. With that one can see that the scale at which the $\alpha_{s}$ is evaluated, is the transverse momentum of the emitted parton. The transverse momentum $k$ is calculated by combining the intrinsic transverse momentum with the transverse momenta emitted in all branchings.

In [11] the fit of PB integrated TMDs to precision measurements of deep inelastic scattering (DIS) cross sections at HERA was performed using xFitter [12]. TMDs obtained this way were used later to obtain predictions for $\mathrm{Z}$ boson $p_{\perp}$ spectrum $[11,13]$. Good agreement with the ATLAS data [14] was obtained. The results in $[11,13]$ were obtained with fixed value of $z_{M}$. In the next section we will investigate the influence of the dynamical $z_{M}$ on the results.

\section{3. iTMDs with angular ordering}

Collinear distributions (reffered to also as the integrated TMDs, iTMDs) can be obtained from eq. (2.1) by integrating the TMD distributions over the transverse momenta ${ }^{2}$

$$
\widetilde{f}_{a}\left(x, \mu^{2}\right)=\int \frac{\mathrm{d}^{2} \boldsymbol{k}}{\pi} \widetilde{A}_{a}\left(x, \boldsymbol{k}, \mu^{2}\right) .
$$

With angular ordering condition, the evolution equation for $\widetilde{f}_{a}$ is given by

$$
\begin{aligned}
& \quad \widetilde{f}_{a}\left(x, \mu^{2}\right)=\Delta_{a}\left(\mu^{2}, \mu_{0}^{2}\right) \widetilde{f}_{a}\left(x, \mu_{0}^{2}\right)+\sum_{b} \int_{\mu_{0}^{2}}^{\mu^{2}} \frac{d \mu^{\prime 2}}{\mu^{\prime 2}} \int_{x}^{1} d z \\
& \times \Theta\left(1-q_{0} / \mu^{\prime}-z\right) \frac{\Delta_{a}\left(\mu^{2}, \mu_{0}^{2}\right)}{\Delta_{a}\left(\mu^{\prime 2}, \mu_{0}^{2}\right)} P_{a b}^{R}\left(z, \alpha_{s}\left((1-z)^{2} \mu^{\prime 2}\right)\right) \widetilde{f}_{b}\left(\frac{x}{z}, \mu^{\prime 2}\right) .
\end{aligned}
$$

One can notice that this formula agrees with Catani-Marchesini-Webber (CMW) result (see eqs. (42), (49) and Sec. 3.4 of [9]). In Ref. [9] this equation was studied at LO with one-loop splitting kernels and running coupling. In PB method it is studied at NLO with two-loop splitting kernels and running coupling.

\section{Mapping evolution scales to transverse momenta}

Exploiting the angular ordering relation $q_{\perp}=(1-z) \mu^{\prime}$, eq. (3.2) can be rewritten by changing the integration variable from the branching scale $\mu^{\prime}$ to the transverse momentum $q_{\perp}$. Depending on the $x$ value, two cases can be distinguished, $x \geq 1-q_{0} / \mu_{0}, 1-q_{0} / \mu_{0}>x>0$ which were studied in detail in [10]. Here we concentrate only on the first case when $1>x \geq 1-q_{0} / \mu_{0}$ for which eq. (3.2) can be written as

$$
\begin{aligned}
\widetilde{f}_{a}\left(x, \mu^{2}\right) & =\Delta_{a}\left(\mu^{2}, \mu_{0}^{2}\right) \widetilde{f}_{a}\left(x, \mu_{0}^{2}\right)+\sum_{b} \int \frac{\mathrm{d} q_{\perp}^{2}}{q_{\perp}^{2}} \int_{x}^{1} \mathrm{~d} z \Theta\left(q_{\perp}^{2}-q_{0}^{2}\right) \Theta\left(\mu^{2}(1-x)^{2}-q_{\perp}^{2}\right) \\
& \times \Theta\left(1-q_{\perp} / \mu-z\right) \frac{\Delta_{a}\left(\mu^{2}, \mu_{0}^{2}\right)}{\Delta_{a}\left(q_{\perp}^{2} /(1-z)^{2}, \mu_{0}^{2}\right)} P_{a b}^{R}\left(z, \alpha_{s}\left(q_{\perp}^{2}\right)\right) \widetilde{f}_{b}\left(\frac{x}{z}, \frac{q_{\perp}^{2}}{(1-z)^{2}}\right) .
\end{aligned}
$$

\footnotetext{
${ }^{2}$ As shown in [6, 7], for $z_{M} \rightarrow 1$ and $\alpha_{S} \rightarrow \alpha_{S}\left(\mu^{\prime 2}\right)$ these are the collinear parton distribution functions satisfying DGLAP evolution equations [15].
} 


\section{Multiple emissions vs single emission}

With this we can compare PB approach with the Kimber-Martin-Ryskin-Watt (KMRW) [16] angular ordered TMD evolution equation:

$$
\widetilde{D}_{a}\left(x, \mu^{2}, q_{\perp}^{2}\right)=T_{a}\left(\mu^{2}, q_{\perp}^{2}\right) \sum_{b} \int_{x}^{1-\frac{q_{\perp}}{q_{\perp}+\mu}} d z P_{a b}^{R}\left(z, \alpha_{s}\left(q_{\perp}^{2}\right)\right) \widetilde{f}_{b}\left(\frac{x}{z}, q_{\perp}^{2}\right)
$$

where the Sudakov form factor is $T_{a}\left(\mu^{2}, q_{\perp}^{2}\right)=\exp \left[-\int_{q_{\perp}^{2}}^{\mu^{2}} \frac{\mathrm{d} q_{\perp}^{\prime 2}}{q_{\perp}^{\prime 2}} \sum_{b} \int_{0}^{1-\frac{q_{\perp}}{q_{\perp}+\mu}} \mathrm{d} z z P_{b a}^{R}\left(z, \alpha_{s}\left(q_{\perp}^{\prime 2}\right)\right)\right]$. The collinear density $\widetilde{f}_{a}\left(x, \mu^{2}\right)$ obeys the evolution equation

$$
\begin{aligned}
\widetilde{f}_{a}\left(x, \mu^{2}\right) & =\widetilde{f}_{a}\left(x, \mu_{0}^{2}\right) T_{a}\left(\mu^{2}, \mu_{0}^{2}\right) \\
& +\int_{\mu_{0}^{2}}^{\left(\frac{\mu(1-x)}{x}\right)^{2}} \frac{d q_{\perp}^{2}}{q_{\perp}^{2}}\left(T_{a}\left(\mu^{2}, q_{\perp}^{2}\right) \sum_{b} \int_{x}^{1-\frac{q_{\perp}}{q_{\perp}+\mu}} d z P_{a b}^{R}\left(z, \alpha_{s}\left(q_{\perp}^{2}\right)\right) \widetilde{f}_{b}\left(\frac{x}{z}, q_{\perp}^{2}\right)\right)
\end{aligned}
$$

To compare PB with KMRW, we neglect the difference between $q_{0}$ and $\mu_{0}$ since this distinction is not used in KMRW approach.

In contrast to PB method, where the transverse momentum is acquired in multiple branchings, the KMRW is a single emission approach: the evolution is performed in one scale up to $q_{\perp}$ and the second scale is generated in the last emission. These two approaches differ also in the scales at which the Sudakov form factors and parton densities $\widetilde{f}_{b}$ are evaluated: KMRW uses just the transverse momentum whereas the PB uses transverse momentum rescaled by $(1-z)$. Moreover, the integration limits are different as well. The KMRW limits lead to the situation when the Sudakov form factor can be larger than 1 losing its probabilistic interpretation and has to be frozen to some value (see e.g [17]).

Interesting remark concerns the Sudakov form factors in these two methods [10]. In the PB approach the Sudakov form factor fulfills the property

$$
\Delta_{a}\left(\mu^{2}, \widetilde{\mu}^{2}\right) \Delta_{a}\left(\widetilde{\mu}^{2}, \mu_{0}^{2}\right)=\Delta_{a}\left(\mu^{2}, \mu_{0}^{2}\right)
$$

for any evolution scale $\widetilde{\mu}$ which is a consequence of its probabilistic interpretation. Also, after changing the integration variables from $\mu^{\prime}$ to $q_{\perp}$, this property remains valid. This is however not the case for KMRW Sudakov form factor.

These two approaches were compared numerically [10] using the TMD sets MRW-CT10nlo [19] which were obtained according to KMRW angular ordering prescription and which can be found in TMDlib and TMDplotter [18]. The same starting distribution CT10nlo [20] was used for both of the approaches for the purpose of the comparison. PB TMD set was obtained with $q_{0}=1 \mathrm{GeV}$.

The result of the comparison is shown in fig. 1 . In the middle $k_{\perp}$ both approaches look very similar: the differences between the two approaches in the parton-density and Sudakov-factor and the phase space compensate for KMRW not taking into account all previous emissions compared to PB. KMRW and PB differ in the low $k_{\perp}$ region where for KMRW we see the intrinsic $k_{\perp}$ constant 
parametrization and for PB the Gaussian intrinsic $k_{\perp}$ smeared during the evolution process. They differ also in the large $k_{\perp}$ region: KMRW has a large $k_{\perp}$ tail coming from their treatment of the Sudakov form factor for $k_{\perp}>\mu$. To explore the effect of single vs multiple emission, PB last step curve was shown in fig. 1 . It was obtained with the same settings as the PB curve, with the only difference in the transverse momentum calculation: in PB last step the transverse momentum is generated only in the last emission. We can see that thanks to multiple emissions the unphysical jump in the distribution coming from matching of the intrinsic $k_{\perp}$ with the evolution is smeared.

It is interesting to look also at the iTMDs shown in fig. 2. In the lower part of the figure the ratios of iTMDs to CT10nlo are plotted. None of the distributions integrate to CT10nlo, what is expected given that the resolution scale $z_{M}$ is far from 1, and the scale of the running coupling $\alpha_{s}$ is $q_{\perp}$. The high- $k_{\perp}$ tail of MRW-CT10nlo results in much higher integrated distribution than other curves.

PB and MRW-CT10nlo TMDs were used to obtain predictions for $\mathrm{Z}$ boson $p_{\perp}$ measurement at $8 \mathrm{TeV}$ [14] shown in fig. 3. We followed the procedure from [11]. We plotted also the prediction obtained with PB TMD Set-2 [11], obtained with fixed $z_{M}$. Since for PB with dynamical $z_{M}$ and KMRW there is no uncertainty band available yet, only the central value for PB TMD Set-2 is shown as well. From the fig. 3 one can clearly see the improvement coming from dynamical resolution scale. MRW-CT10nlo does not describe neither the high $p_{\perp}$ region nor the low $p_{\perp}$ slope.

\section{Conclusions}

In this work we discussed the PB TMD evolution equation with angular ordering condition including dynamical soft gluon resolution scale $z_{M}$. Thanks to the dynamical $z_{M}$, we were able to establish connection of PB with other well known approaches, CMW and KMRS. We discussed the impact of the dynamical soft gluon resolution scale on the precise predictions of $\mathrm{Z}$ boson $p_{\perp}$ and we showed improvement compared to using fixed $z_{M}$ value.

Acknowledgments The results presented in this article were obtained in collaboration with Francesco Hautmann, Aron Mees van Kampen and Lissa Keersmaekers. We thank Hannes Jung for many discussions.

\section{References}

[1] J. C. Collins, D. E. Soper and G. F. Sterman, Adv.Ser. Direct. High Energy Phys. 5 (1989) 1

[2] R. Angeles-Martinez et al., Acta Phys. Polon. B 46 (2015) no.12, 2501

[3] F. Hautmann and H. Jung, Eur. Phys. J. C 72, 2254 (2012)

S. Dooling, P. Gunnellini, F. Hautmann and H. Jung, Phys. Rev. D 87, no. 9, 094009 (2013)

[4] S. Catani, M. Ciafaloni and F. Hautmann, Phys. Lett. B 242 (1990) 97

S. Catani, M. Ciafaloni and F. Hautmann, Nucl. Phys. B 366 (1991) 135

[5] H. Jung and G. P. Salam, Eur. Phys. J. C 19, 351 (2001)

[6] F. Hautmann, H. Jung, A. Lelek, V. Radescu and R. Zlebcik, Phys. Lett. B 772 (2017) 446

[7] F. Hautmann, H. Jung, A. Lelek, V. Radescu and R. Zlebcik, JHEP 1801 (2018) 070 


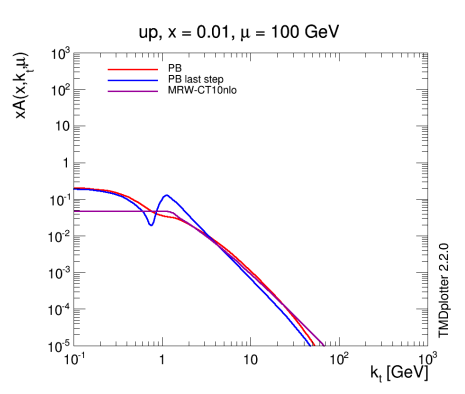

Figure 1: TMDs from $\mathrm{PB}$ and Figure 2: The results of integratKMRW as functions of transverse ing TMDs over all $k_{\perp}$ as functions momentum for up quark at $x=$ of $x$. 0.01 and $\mu=100 \mathrm{GeV}$.

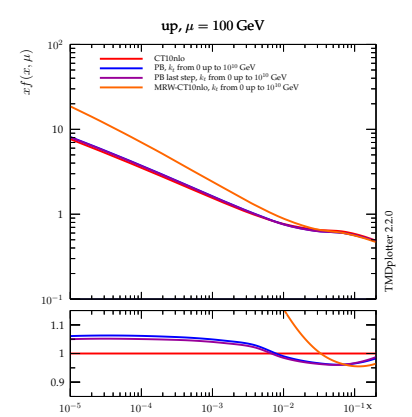

[8] G. Marchesini and B. R. Webber, Nucl. Phys. B 238, 1 (1984).

S. Catani, B. R. Webber and G. Marchesini, Nucl. Phys. B 349, 635 (1991).

[9] G. Marchesini and B. R. Webber, Nucl. Phys. B 310, 461 (1988).

[10] F. Hautmann, L. Keersmaekers, A. Lelek and A. M. Van Kampen, arXiv:1908.08524

[11] A. Bermudez Martinez, P. Connor, H. Jung, A. Lelek, R. Zlebcik, F. Hautmann and V. Radescu, Phys. Rev. D 99 (2019) no.7, 074008

[12] S. Alekhin et al., Eur. Phys. J. C 75 (2015) no.7, 304

[13] A. Bermudez Martinez et al., Phys. Rev. D 100, 074027 (2019)

[14] G. Aad et al. [ATLAS Collaboration], Eur. Phys. J. C 76 (2016) no.5, 291

[15] V. N. Gribov and L. N. Lipatov, Sov. J. Nucl. Phys. 15 (1972) 438

L. N. Lipatov, Sov. J. Nucl. Phys. 20 (1975) 94

G. Altarelli and G. Parisi, Nucl. Phys. B 126 (1977) 298

Y. L. Dokshitzer, Sov. Phys. JETP 46 (1977) 641

[16] M. A. Kimber, A. D. Martin and M. G. Ryskin, Eur. Phys. J. C 12 (2000) 655

A. D. Martin, M. G. Ryskin and G. Watt, Eur. Phys. J. C 66 (2010) 163

[17] K. Golec-Biernat and A. M. Stasto, Phys. Lett. B 781, 633 (2018)

[18] F. Hautmann, H. Jung, M. Kramer, P. J. Mulders, E. R. Nocera, T. C. Rogers and A. Signori, Eur. Phys. J. C 74 (2014) 3220

[19] M. Bury, A. van Hameren, H. Jung, K. Kutak, S. Sapeta and M. Serino, Eur. Phys. J. C 78 (2018) no. 2,137

[20] H. L. Lai, M. Guzzi, J. Huston, Z. Li, P. M. Nadolsky, J. Pumplin and C.-P. Yuan, Phys. Rev. D 82, 074024 (2010) 